\title{
Concentrated dark matter: Enhanced small-scale structure from codecaying dark matter
}

\author{
Jeff A. Dror, ${ }^{1,2, *}$ Eric Kuflik, ${ }^{3, \dagger}$ Brandon Melcher, ${ }^{4, \$}$ and Scott Watson ${ }^{4,}$ \\ ${ }^{1}$ Department of Physics, University of California, Berkeley, California 94720, USA \\ ${ }^{2}$ Ernest Orlando Lawrence Berkeley National Laboratory, University of California, \\ Berkeley, California 94720, USA \\ ${ }^{3}$ Racah Institute of Physics, Hebrew University of Jerusalem, Jerusalem 91904, Israel \\ ${ }^{4}$ Department of Physics, Syracuse University, Syracuse, New York 13244, USA
}

(Received 18 November 2017; revised manuscript received 26 January 2018; published 22 March 2018)

\begin{abstract}
We study the cosmological consequences of codecaying dark matter-a recently proposed mechanism for depleting the density of dark matter through the decay of nearly degenerate particles. A generic prediction of this framework is an early dark matter dominated phase in the history of the Universe, that results in the enhanced growth of dark matter perturbations on small scales. We compute the duration of the early matter dominated phase and show that the perturbations are robust against washout from free streaming. The enhanced small-scale structure is expected to survive today in the form of compact microhalos and can lead to significant boost factors for indirect-detection experiments, such as FERMI, where dark matter would appear as point sources.
\end{abstract}

DOI: 10.1103/PhysRevD.97.063524

\section{INTRODUCTION}

The thermal history of the Universe is established below temperatures around a MeV. Through the precise predictions and measurements employed to study big bang nucleosynthesis (BBN) and the cosmic microwave background, we can now place stringent bounds on any particles beyond the Standard Model (SM) that were in thermal equilibrium at these times. Conversely, the SM does not provide a means of probing its thermal history at temperatures higher than $\mathcal{O}(\mathrm{MeV})$, leading to the common lore that any thermal dynamics above this scale will not be accessible to experiment. Many well-motivated models of dark matter (DM) predict masses above this scale; this makes it challenging to use cosmology to place constraints on the creation mechanism of DM.

Recently, however, it has been proposed that an early period of matter domination (before BBN) would have observable implications [1-3]. The idea is that a period of early matter domination would lead to structure formation in DM prior to freeze-out. These early seeds of structure

\footnotetext{
*jdror@lbl.gov

eric.kuflik@mail.huji.ac.il

tbsmelche@syr.edu

§swatson@syr.edu
}

Published by the American Physical Society under the terms of the Creative Commons Attribution 4.0 International license. Further distribution of this work must maintain attribution to the author(s) and the published article's title, journal citation, and DOI. Funded by SCOAP. can result in concentrated dark matter, where the bulk of dark matter is found in dark compact objects today. This intriguing possibility has led to a surge of studies of early structure formation [2-9]. However, while these models do predict significant matter domination and pre-BBN structure formation, one often finds that the perturbations do not survive to today. This is either due to DM being kinetically coupled to the radiation bath during or after the matter dominated epoch or because reheating washes out the perturbations.

An alternative mechanism which leads to an early matter dominated era and enhanced structure formation is the recently proposed "codecaying DM" framework [10]. (For other recent models that include an early period of matter domination in a dark sector, see Refs. [11-14].) Here, DM itself comes to dominate the total energy density, leading to the creation of small-scale structure. Part of the dark sector later decays to SM particles, reheating the SM bath prior to BBN. In this paper, we show that since codecaying DM decouples from all lighter degrees of freedom very early in the history of the Universe, the substructure is not washed out by free-streaming or reheating effects. This results in a viable candidate for significantly enhanced small-scale substructure from early Universe matter domination.

\section{CODECAYING DARK MATTER}

We begin with a brief review of codecaying DM, referring the reader to Ref. [10] for more details. The lightest particles in the dark sector are a (nearly) degenerate 
species of dark particles, denoted by $A$ and $B$, where $A$ will comprise the DM today and $B$ is unstable, decaying out of equilibrium. The dark particles thermally decouple from the SM, while they are still relativistic in the early Universe. The two dark sector particles remain in equilibrium with each other via large $A A \leftrightarrow B B$ annihilations, but due to being degenerate and decoupled from the SM, they do not undergo Boltzmann suppression as they become nonrelativistic. Instead, the suppression of the number density occurs when the $B$ particles begin to decay, which results in depletion of the $A$ population. Eventually, the $A$ population drops out of thermal contact with the $B$ 's, and $A$ abundance freezes out. We note that codecaying dark matter exists naturally in theories where there is an approximate symmetry between the two states which is broken to induce a decay to the SM. One possibility is to use a remnant of a broken gauge symmetry as was considered in Ref. [10], though there are other possibilities and exploring the model space is an ongoing effort.

When the dark sector fields become nonrelativistic, the dark and visible sectors can be described as a system of interacting fluids. The background evolution equations for the energy densities are

$$
\begin{gathered}
\rho_{A}^{\prime}+\rho_{B}^{\prime}=-3\left(\rho_{A}+\rho_{B}\right)-\frac{\Gamma_{B}}{H} \rho_{B}, \\
\rho_{A}^{\prime}=-3 \rho_{A}-\frac{\langle\sigma v\rangle}{m H}\left[\rho_{A}^{2}-\rho_{B}^{2}\right], \\
\rho_{r}^{\prime}=-4 \rho_{r}+\frac{\Gamma_{B}}{H} \rho_{B},
\end{gathered}
$$

where $\rho_{\alpha}$ is the energy density of the respective particles and $r$ refers to the SM bath. We use primes to denote derivatives with respect to the number of $e$-folds, $m$ is the mass of $A, B$, and $\langle\sigma v\rangle$ is the thermally averaged cross section for $A A \rightarrow B B$. For $s$-wave scattering, we can parametrize the thermally averaged cross section in terms of the zero temperature cross section, $\sigma,\langle\sigma v\rangle \simeq$ $\sigma \sqrt{(16 / \pi)\left(T_{A, B} / m\right)}$. The unique dependence on the temperature of the dark sector, $T_{A, B}$, can lead to novel indirectdetection signatures $[10,15,16]$.

In this work, we will assume that dark number changing processes are small and that "cannibalization" [17] is absent. In actual models, cannibalization may play a role, even if very minor. We leave the study of the effects of cannibalization on structure growth to future work [18]. Furthermore, depending on the model realizing the codecay framework, the dark sector may not have a well-defined temperature slightly prior to chemical decoupling, which could affect the computation of the relic density (see Refs. [19,20] for related work). However, such model dependencies are beyond the scope of this work.

The solution to the background energy densities in Eqs. (1)-(3) is shown in the top panel of Fig 1. For a representative benchmark point with significant matter domination, we take $m=100 \mathrm{GeV}, \Gamma_{B}=10^{-22} \mathrm{GeV}$, and a ratio of the number of degrees of freedom in the dark sector to the SM at kinetic decoupling to be $\xi=0.1$. This scenario corresponds to approximately nine $e$-folds of matter domination.

From the time the DM becomes nonrelativistic, its density redshifts like matter and quickly comes to dominate the energy density of the Universe (marked by $N_{\mathrm{MD}}$ in Fig. 1). Later, $B$ begins to decay into the SM, reheating the SM bath, and shortly afterward the Universe returns to radiation domination (marked by $N_{\mathrm{RH}}$ in Fig. 1). The period of (dark) matter domination can span many $e$-folds, during which density perturbations will grow linearly. The number of $e$-folds of matter domination is

$N_{\mathrm{RH}}-N_{\mathrm{MD}} \equiv \log \frac{a_{\mathrm{RH}}}{a_{\mathrm{MD}}} \sim \log \frac{a_{\Gamma}}{a_{\mathrm{MD}}} \simeq \frac{1}{3} \log \frac{n_{\mathrm{A}+\mathrm{B}, \mathrm{MD}}}{n_{A+B, \Gamma}}$.

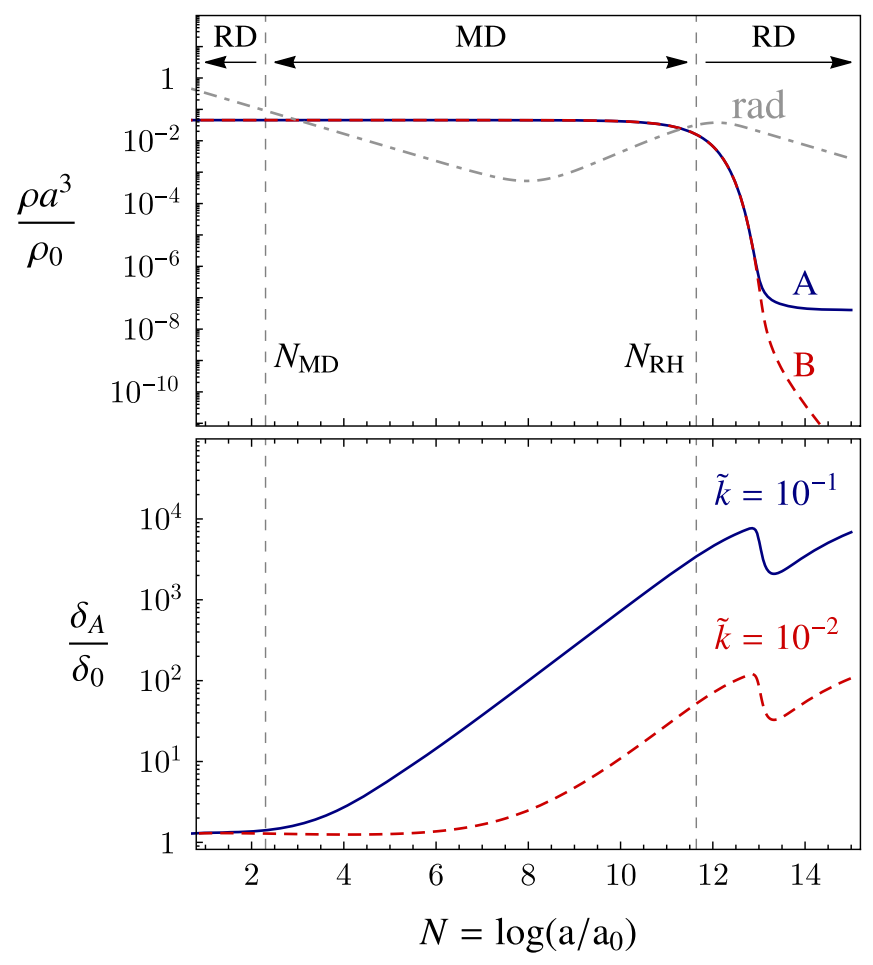

FIG. 1. Top: Evolution of the background energy densities relative to the total initial density for the benchmark point, $m=100 \mathrm{GeV}, \Gamma_{B}=10^{-22} \mathrm{GeV}$, and $\xi=0.1$. The subscript 0 , refers to values at $N=0$, corresponding to where the dark matter becomes nonrelativistic $\left(T_{A, B}=m\right)$. Dark matter quickly comes to dominate the energy density of the Universe, which lasts until slightly after the onset of $B$ decay. Bottom: Evolution of the dark sector density perturbation for modes $\tilde{k} \equiv k / H_{N=0}=10^{-1}, 10^{-2}$, which enters the horizon during matter domination. The modes grows linearly during matter domination, dips during freeze-out, and then grows logarithmically during the radiation dominated epoch. 
The subscripts $\Gamma$ and MD denote values evaluated when $\Gamma_{B}=H$ and at the onset of matter domination, respectively. In the first step, we assumed that most of matter domination occurs between the onset of matter domination and the beginning of decay (it roughly takes one $e$-fold for the decay to convert most of the DM into radiation). In the second step, we used the fact that the number of dark matter particles is approximately conserved until decays begin. The expression can be further simplified using the relations at the onset of matter domination, $n_{\mathrm{A}+\mathrm{B}, \mathrm{MD}} \sim m^{3}$, and at decay, $\Gamma_{B}^{2} \simeq H_{\Gamma}^{2} \sim m n_{A+B} / M_{\mathrm{pl}}$. This gives

$$
N_{\mathrm{RH}}-N_{\mathrm{MD}} \simeq \frac{1}{3} \log \frac{m^{4}}{M_{\mathrm{pl}}^{2} \Gamma_{B}^{2}}+\mathcal{O}(1)
$$

In Fig. 2, we show the parameter space of codecaying $\mathrm{DM}$ in the $\left(m, \Gamma_{B}\right)$ plane where the cross section is fixed to get the correct relic abundance. Overlayed are the number of $e$-folds of matter domination, given that the two sectors were once kinetically coupled early in the Universe and had entropy ratio $\xi=0.1$ at kinetic decoupling [suppressed in Eq. (5) is an additional logarithmic dependence on $\xi$ ]. For this choice $\xi$, up to $22 e$-folds of matter domination are possible. Larger values of $\xi$ would increase the duration of the early matter dominated epoch.

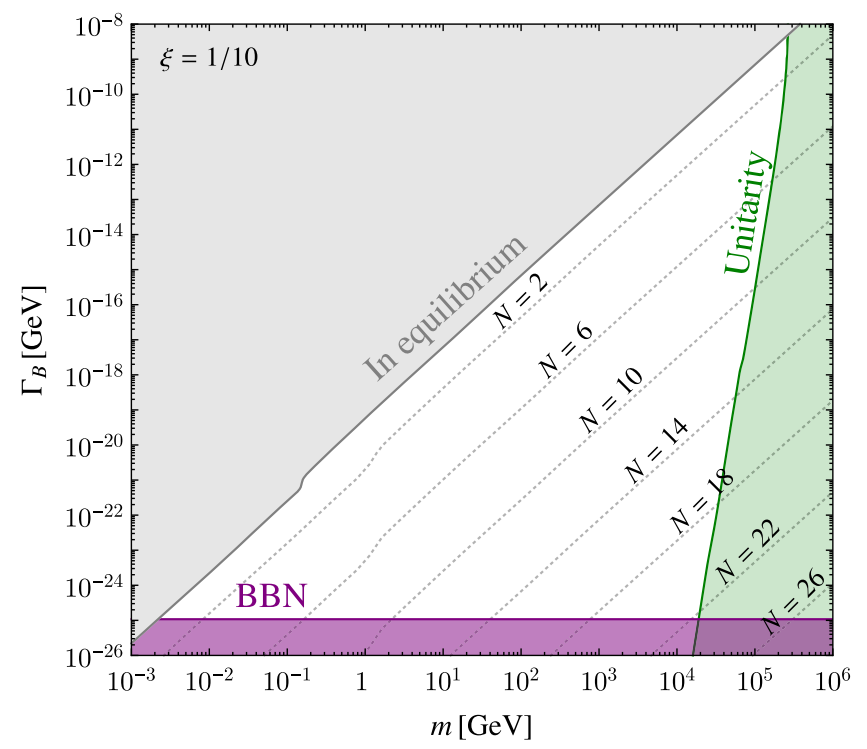

FIG. 2. The parameter space for codecaying DM assuming no cannibalization that yields the correct relic abundance [10]. The different regions show constraints from $\Delta N_{\text {eff }}$ (purple); ensuring that DM decays out of equilibrium with the SM (and can come to dominate the energy density) (gray); and unitarity constraints (green). In addition, there exist model-dependent constraints from indirect-detection searches, which we omit here. The number of $e$-folds of matter domination are overlaid, where here $N$ denotes, in shorthand, $N_{\mathrm{RH}}-N_{\mathrm{MD}}$.

\section{GROWTH OF COSMOLOGICAL PERTURBATIONS AND FREE STREAMING}

We now explore the effects of early matter domination on the growth of codecaying DM perturbations, where they are expected to grow rapidly. In longitudinal gauge, without anisotropic stress, the metric is

$$
d s^{2}=-(1+2 \Phi) d t^{2}+a(t)^{2}(1-2 \Phi) \delta_{i j} d x^{i} d x^{j}
$$

Working in momentum space, the time-time component of the perturbed Einstein equation is

$$
\left(\frac{k^{2}}{3 a^{2} H^{2}}+1\right) \Phi+\Phi^{\prime}=-\frac{1}{6 H^{2} m_{p}^{2}} \sum_{\alpha} \delta \rho_{(\alpha)},
$$

where $v_{(\alpha)}, \delta \rho_{(\alpha)}$, and $\delta p_{(\alpha)}$ are scalar velocity, density, and pressure perturbations for each fluid, respectively. Introducing the fractional density perturbations $\delta_{(\alpha)} \equiv$ $\delta \rho_{(\alpha)} / \rho_{(\alpha)}$ and defining the velocity perturbation for each fluid as $\theta_{(\alpha)}=a^{-1} \nabla^{2} v_{(\alpha)}$, the continuity equations in momentum space are given by

$$
\begin{aligned}
\delta_{A}^{\prime} & +\frac{\theta_{A}}{a H}-3 \Phi^{\prime} \\
& =-\frac{\langle\sigma v\rangle}{m H \rho_{A}}\left[\rho_{A}^{2}\left(\Phi+\delta_{A}\right)-\rho_{B}^{2}\left(\Phi+2 \delta_{B}-\delta_{A}\right)\right],
\end{aligned}
$$

$$
\begin{aligned}
\delta_{B}^{\prime} & +\frac{\theta_{B}}{a H}-3 \Phi^{\prime} \\
& =-\frac{\Gamma_{B}}{H} \Phi+\frac{\langle\sigma v\rangle}{m H \rho_{B}}\left[\rho_{A}^{2}\left(\Phi+2 \delta_{A}-\delta_{B}\right)-\rho_{B}^{2}\left(\Phi+\delta_{B}\right)\right],
\end{aligned}
$$

$$
\delta_{r}^{\prime}+\frac{4}{3} \frac{\theta_{r}}{a H}-4 \Phi^{\prime}=\frac{\Gamma_{B}}{H} \frac{\rho_{B}}{\rho_{r}}\left[\Phi+\delta_{B}-\delta_{r}\right] .
$$

Similarly, the equations for the velocity perturbations are

$$
\begin{aligned}
& \theta_{A}^{\prime}+\theta_{A}-\frac{k^{2}}{a H} \Phi=\frac{\langle\sigma v\rangle}{m H \rho_{A}}\left[\rho_{B}^{2}\left(\theta_{B}-\theta_{A}\right)\right], \\
& \theta_{B}^{\prime}+\theta_{B}-\frac{k^{2}}{a H} \Phi=\frac{\langle\sigma v\rangle}{m H \rho_{B}}\left[\rho_{A}^{2}\left(\theta_{A}-\theta_{B}\right)\right], \\
& \theta_{r}^{\prime}-\frac{k^{2}}{a H}\left(\frac{\delta_{r}}{4}+\Phi\right)=\frac{\Gamma_{B}}{H} \frac{\rho_{B}}{\rho_{r}}\left[\frac{3}{4} \theta_{B}-\theta_{r}\right] .
\end{aligned}
$$

We took each fluid to have a definite equation of state with pressure $p_{(\alpha)}=w_{(\alpha)} \rho_{(\alpha)}$ and hence $\delta p_{(\alpha)}=c_{s(\alpha)}^{2} \delta \rho_{(\alpha)}$ with $c_{s(A)}^{2}=c_{s(B)}^{2}=0, \quad c_{s(r)}^{2}=1 / 3$. This set of differential 
equations can be closed using Eq. (7). ${ }^{1}$ We take adiabatic initial conditions ${ }^{2}$ for the perturbations as in Refs. [2,4].

Our solutions for the linear density perturbations are presented in the bottom panel of Fig. 1. Here, we consider modes $k=0.1 \times H_{N=0}$ and $k=0.01 \times H_{N=0}$, which enter the horizon at $N \simeq 2.7$ and $N \simeq 6.7$, respectively. After entering the horizon, modes grows linearly during matter domination, until reheating $\left(N_{R H} \simeq 13\right)$. At this point, $A$ begins to freeze out. The $A$ and $B$ particles are no longer in chemical equilibrium, so the annihilations can change the density contrast [see Eq. (8)]; $A A \rightarrow B B$ annihilations can annihilate away $A$ overdensities into $B$ particles, which then promptly decay. This results in the dip in Fig. 1. As $A$ completely freezes out, number changing processes stop, the comoving density is fixed, and the perturbations grow logarithmically during the radiation dominated era.

The amplitude of general DM perturbations can be estimated analytically by approximating the decay as instantaneous and the dark matter as a single fluid. Perturbations that enter the Hubble radius prior to reheating $\left(k>k_{\mathrm{RH}}\right)$ take the form

$$
\left|\delta_{A}\right|=\frac{2}{3}\left(\frac{k}{k_{\mathrm{RH}}}\right)^{2} \Phi_{0}\left[1+\ln \left(\frac{a}{a_{\mathrm{RH}}}\right)\right],
$$

where $k_{\mathrm{RH}}$ is given by

$k_{\mathrm{RH}} \equiv a_{\mathrm{RH}} H_{\mathrm{RH}}=0.1 \mathrm{pc}^{-1} \times\left(\frac{T_{\mathrm{RH}}}{3 \mathrm{MeV}}\right)\left(\frac{g_{*}\left(T_{\mathrm{RH}}\right)}{10.75}\right)^{1 / 6}$,

with $T_{\mathrm{RH}}$ the temperature of the SM bath immediately proceeding decay. Equation (14) is found by accounting for the initial amplitude of the perturbations as a mode enters the horizon, which grows linearly during matter domination and then logarithmically with the scale factor after reheating. We note that the above expressions agree with those of Ref. [3].

The scale of reheating, given by Eq. (15), should be compared to the scales of kinetic decoupling and free streaming, since both can wash out structure. Early kinetic decoupling is a defining feature of the framework, and therefore there is no collisional damping to suppress growth of structure in codecaying DM. On the other hand, the freestreaming wavelength is given by

\footnotetext{
${ }^{1}$ In principle, the inevitable dark temperature dependence of the thermally averaged cross section requires the inclusion of an additional first order perturbation equation. Since the dark temperature around freeze-out may not be well understood in some models realizing the codecay framework, we do not include these effects here but emphasize the schematic nature of the numerical solutions. Furthermore, the annihilations terms only slightly affect the perturbations, and thus the inclusion dark temperature perturbations will have a small effect on the final results. We have checked this explicitly.

${ }^{2}$ For a situation with nonadiabatic initial conditions in a matter phase, we refer to Ref. [21].
}

$$
\frac{1}{k_{\mathrm{FS}}}=\int_{a_{\mathrm{kd}}}^{a_{\mathrm{eq}}} \frac{\langle v\rangle}{H a^{2}} d a
$$

where $\langle v\rangle$ is the average velocity of the dark $A+B$ fluid and $a_{\mathrm{kd}}$ and $a_{\mathrm{eq}}$ are the scale factors at kinetic decoupling and (the late time) matter-radiation equality, respectively. Immediately after kinetic decoupling from the SM, the freestreaming dark matter fluid is relativistic, $\langle v\rangle=1$. Then, close to when the $\mathrm{U}$ becomes matter dominated, the DM becomes nonrelativistic, and its velocity begins to redshift like the scale factor, and quickly slows. Thus, most of the free streaming takes place before the matter dominated phase begins. Therefore, for codecaying DM, the freestreaming length is roughly the size of the horizon at the time of matter domination, $k_{\mathrm{FS}} \simeq k_{\mathrm{MD}}$. Free streaming will only potentially wash out modes that have just entered the horizon after matter domination.

Relative to the scale at reheating,

$$
\frac{k_{\mathrm{FS}}}{k_{\mathrm{RH}}} \simeq \frac{k_{\mathrm{MD}}}{k_{\mathrm{RH}}} \simeq\left(\frac{g_{*, \mathrm{MD}}}{g_{*, \mathrm{RH}}}\right)^{1 / 6}\left(\frac{m \xi}{T_{\mathrm{RH}}}\right)^{2 / 3} .
$$

The free-streaming damping scale will set the critical scale $k_{\mathrm{cut}}=k_{\mathrm{MD}}$ for the smallest size of subhalos. In Ref. [2], it was found that whichever scale sets the cutoff $k_{\text {cut }}$ (in general $k_{\mathrm{FS}}$ or $k_{\mathrm{KD}}$, whichever is smaller) needs only to satisfy $k_{\mathrm{cut}} / k_{\mathrm{RH}} \gtrsim 10$ in order for the early matter epoch to lead to enhanced small-scale structures (microhalos). In codecaying DM, this is typically orders of magnitude larger [e.g., $\mathcal{O}\left(10^{3}\right)$ for the parameters in Fig. 1].

The survival of small-scale structure in codecaying DM is in contrast to the case of dark matter produced from the decays of an out-of-equilibrium particle, such as moduli decaying to a neutralino. Indeed, in Ref. [4], it was shown in this context that the enhanced growth of structure would not survive either due to late time kinetic decoupling or free streaming of relativistic DM produced from moduli decays.

\section{PRESENT-DAY SUBSTRUCTURE}

Having established that the enhanced perturbations for the growth of microhalos can survive reheating and washout effects, we now consider the possible implications for structure formation today. Following Refs. [2,3], which use the Press-Schechter formalism [22], we now comment on the qualitative features of the predicted substructure.

References [2,3] established the fraction of the dark matter abundance found in microhalos today resulting from an early matter dominated phase. There, it was found that the effect of the enhanced growth can be captured by altering the transfer function on the relevant scales, namely between the beginning of matter domination and the time of reheating. Additionally, the growth function will differ from the standard scenario since baryons will not play a role in structure formation at these scales. 
From the Press-Schechter formalism, we expect that once the rms density perturbation exceeds the critical value $\delta_{c}=1.69$ compact microhalos will form. References [2,3] found that the rms density perturbation is rather insensitive to the reheat temperature but depends critically on $k_{\mathrm{cut}} / k_{\mathrm{RH}}$. If this ratio exceeds $\mathcal{O}(10)$, then microhalos can form; importantly, this ratio is orders of magnitudes larger in codecaying DM. In particular, it was shown that this ratio not only determines the masses of the microhalos but also their time of formation. As the $k_{\mathrm{cut}} / k_{\mathrm{RH}}$ ratio increases, the redshift at which these microhalos form increases as well. Moreover, the higher the value of the ratio, the more peaked the mass distribution of the microhalos is toward the largest possible size. The largest microhalos are set by the size of the horizon at reheating, $1 / k_{\mathrm{RH}}$, and will have masses smaller than or near

$M_{\mathrm{RH}} \equiv \frac{4}{3} \pi \rho_{A}^{(0)} k_{\mathrm{RH}}^{-3} \simeq 10^{3} M_{\oplus}\left(\frac{3 \mathrm{MeV}}{T_{\mathrm{RH}}}\right)^{3}\left(\frac{10.75}{g_{*}\left(T_{\mathrm{RH}}\right)}\right)^{1 / 2}$.

Namely, for reheating near BBN, codecaying DM can produce microhalos with masses as large as a thousand Earth masses or less. The combination of the above with the natural prediction in the codecaying DM framework of $k_{\text {cut }} / k_{\mathrm{RH}} \gg 1$ suggests that codecaying DM will lead to the formation of microhalos with masses peaking around the value given in Eq. (18).

If these structures survive until today, they lead to high concentrations of DM which result in large boost factors for the self-annihilation of DM [3]. Thus, codecaying DM predicts enhanced signals in indirect-detection experiments.
Furthermore, subhalos of the size predicted here would appear as point sources and could give a DM explanation [23] to the unidentified point sources observed by FERMILAT [24-26].

We conclude with several comments and open questions. First, N-body simulations are most likely needed in order to evaluate the survival rates of the microhalos from high redshifts until today. Next, it would be interesting to study the internal structure of these halos. Finally, the early matter dominated phase can also result in the formation of primordial black holes [27,28], which can provide another component of the cosmological DM, in addition to the codecaying DM. We leave the exploration of these important questions to future work.

\section{ACKNOWLEDGMENTS}

We thank Avishai Dekel, Cosmin Isle, Adrienne Erickcek, Jonah Kudler-Flam, Michael Geller, and Gustavo Marques Tavares for useful discussions and Yonit Hochberg for comments on the manuscript. S. W. and B. M. were supported in part by NASA Astrophysics Theory Grant No. NNH12ZDA001N and DOE Grant No. DE-FG02-85ER40237. E. K. is supported by the I-CORE Program of the Planning Budgeting Committee (Grant No. 1937/12), the Israel Science Foundation (Grant No. 1111/17), and the Binational Science Foundation (Grant No. 2016153). E. K. and S. W. are thankful for the hospitality of the Aspen Center for Physics, which is supported by NSF Grant No. PHY-1066293. J. A. D. is supported in part by the DOE under Contract No. DEAC02-05CH11231.
[1] G. B. Gelmini and P. Gondolo, J. Cosmol. Astropart. Phys. 10 (2008) 002.

[2] A. L. Erickcek and K. Sigurdson, Phys. Rev. D 84, 083503 (2011).

[3] A. L. Erickcek, Phys. Rev. D 92, 103505 (2015).

[4] J. Fan, O. Ozsoy, and S. Watson, Phys. Rev. D 90, 043536 (2014).

[5] K.-Y. Choi and T. Takahashi, Phys. Rev. D 96, 041301 (2017).

[6] O. Ozsoy, Ph. D. thesis, Syracuse University, 2017.

[7] I. R. Waldstein, A. L. Erickcek, and C. Ilie, Phys. Rev. D 95, 123531 (2017).

[8] G. Barenboim and J. Rasero, J. High Energy Phys. 04 (2014) 138.

[9] J. T. Giblin, G. Kane, E. Nesbit, S. Watson, and Y. Zhao, Phys. Rev. D 96, 043525 (2017).
[10] J. A. Dror, E. Kuflik, and W. H. Ng, Phys. Rev. Lett. 117, 211801 (2016).

[11] L. Randall, J. Scholtz, and J. Unwin, J. High Energy Phys. 03 (2016) 011.

[12] D. Pappadopulo, J. T. Ruderman, and G. Trevisan, Phys. Rev. D 94, 035005 (2016).

[13] M. Farina, D. Pappadopulo, J. T. Ruderman, and G. Trevisan, J. High Energy Phys. 12 (2016) 039.

[14] A. Berlin, D. Hooper, and G. Krnjaic, Phys. Rev. D 94, 095019 (2016).

[15] J. Kopp, J. Liu, T. R. Slatyer, X.-P. Wang, and W. Xue, J. High Energy Phys. 12 (2016) 033.

[16] S. Okawa, M. Tanabashi, and M. Yamanaka, Phys. Rev. D 95, 023006 (2017).

[17] E. D. Carlson, M. E. Machacek, and L. J. Hall, Astrophys. J. 398, 43 (1992). 
[18] J. Dror, E. Kuflik, B. Melcher, and S. Watson (to be published).

[19] R. T. D’Agnolo, D. Pappadopulo, and J. T. Ruderman, Phys. Rev. Lett. 119, 061102 (2017).

[20] T. Binder, T. Bringmann, M. Gustafsson, and A. Hryczuk, Phys. Rev. D 96, 115010 (2017).

[21] L. Iliesiu, D. J. E. Marsh, K. Moodley, and S. Watson, Phys. Rev. D 89, 103513 (2014).

[22] W. H. Press and P. Schechter, Astrophys. J. 187, 425 (1974).

[23] P. Agrawal and L. Randall, J. Cosmol. Astropart. Phys. 12 (2017) 019.
[24] S. K. Lee, M. Lisanti, B. R. Safdi, T. R. Slatyer, and W. Xue, Phys. Rev. Lett. 116, 051103 (2016).

[25] R. Bartels, S. Krishnamurthy, and C. Weniger, Phys. Rev. Lett. 116, 051102 (2016).

[26] M. Ajello et al. (Fermi-LAT Collaboration), arXiv:1705 .00009.

[27] J. Georg and S. Watson, J. High Energy Phys. 09 (2017) 138.

[28] J. Georg, G. Sengor, and S. Watson, Phys. Rev. D 93, 123523 (2016). 